\title{
Hacia una historia de la "Cueva de los Guanacos". Una visión diacrónica del arte rupestre de Oyola 34 (Catamarca)
}

Eugenia Ahets Etcheberry*

\author{
Fecha de defensa: 30 de abril de 2020 \\ Director: Dr. Lucas Ignacio Gheco \\ Jurados: Dras. Mara Basile y Dánae Fiore
}

Desde mediados del siglo XX, la sierra de El Alto-Ancasti (al este de la provincia de Catamarca) cautivó la atención de las investigaciones arqueológicas a raíz de los numerosos abrigos con arte rupestre que se distribuyen en su ladera oriental boscosa. Tradicionalmente, estos trabajos adscribieron las pinturas a una única cultura -La Aguada- o período dentro del desarrollo cultural del noroeste argentino -período Medio o de Integración Regional- y les otorgaron una función mágicoreligiosa o ritual (De la Fuente, 1979; González, 1977; Segura, 1988). Recientes estudios han problematizado estas atribuciones uniformes señalando sus dificultades para dar cuenta de la heterogeneidad que caracteriza al arte rupestre de la zona, al mismo tiempo que destacaron la necesidad de indagar en los procesos históricos locales que produjeron, transformaron y se desarrollaron en estos paisajes (Gheco, 2017; Gordillo, Zuccarelli y Eguia, 2017; Quesada, Gastaldi y Granizo, 2012). Esta tesis se propone contribuir al conocimiento de dichos procesos, retomando los trabajos realizados en el sitio arqueológico de Oyola y tensionando algunas de las interpretaciones planteadas a partir de un nuevo caso de estudio: el abrigo Oyola 34, también conocido localmente como la "Cueva de los guanacos".

Ubicado en el sector meridional de la sierra y próximo al poblado homónimo, el sitio arqueológico de Oyola está conformado por, al menos, 38 cuevas y aleros con arte rupestre. Durante los últimos 10 años, parte de las investigaciones desarrolladas allí han indagado en los procesos históricos de producción, uso y transformación de los abrigos pintados y han delineado dos tendencias generales para interpretarlos (Gheco, 2017). La primera de ellas señala que la mayoría de las cuevas y aleros seleccionados para confeccionar los motivos rupestres presenta dimensiones espaciales pequeñas, configurando experiencias íntimas y estáticas de observación/ acción. La segunda se asocia con la homogeneidad estilística y cromática que se observa en las figuras de cada abrigo y la baja frecuencia de superposiciones entre ellas, características que dan a los paneles una apariencia inalterada a pesar de que fueron elaborados y modificados a lo largo del tiempo. Ahora bien, aunque en términos mayoritarios ambas tendencias son aplicables a gran parte de los abrigos del sitio, algunas

* Instituto de Investigaciones sobre el Patrimonio Cultural, Universidad Nacional de San Martín - CONICET. B. Quinquela Martín 1784 (CP C1296ADJ), Ciudad Autónoma de Buenos Aires, Argentina. E-mail: eahets@gmail.com cuevas presentan características que se apartan de ellas, ya sea por sus mayores tamaños, diversidad cromática o número de superposiciones. En la medida en que se trata de casos minoritarios, entendemos que no anulan las conclusiones previas, sino que constituyen tensiones a las interpretaciones realizadas que resaltan la necesidad de comprender en detalle estas historias particulares para continuar complejizando el conocimiento sobre el arte rupestre del sitio.

Oyola 34 constituye uno de dichos casos excepcionales. Más específicamente, esta cueva se aparta de las tendencias generales propuestas para el sitio, en primer lugar, por sus mayores dimensiones espaciales: está conformada por tres cámaras, la principal de las cuales (en tamaño y número de motivos rupestres) tiene $20 \mathrm{~m}^{2}$ de superficie y $3 \mathrm{~m}$ de altura, aproximadamente (Figura 1).

En segundo lugar, su excepcionalidad reside en que las 49 figuras documentadas allí se destacan por su diversidad y buen estado de conservación: han sido confeccionadas en colores negros, naranjas y blancos, y mayoritariamente parecen referir a camélidos, aunque de distintos tamaños, morfologías, tonalidades y ubicaciones espaciales (Figura 2).

Considerando estas particularidades, ¿en qué medida Oyola 34 puede ser comprendida a partir de las investigaciones realizadas en el resto de los abrigos del sitio?, ¿cuáles podrían ser las causas de sus características aparentemente excepcionales?, ¿qué implicancias tiene esto para con las interpretaciones del arte rupestre de Oyola propuestas hasta el momento? Partiendo de estos interrogantes, el objetivo general que orientó la investigación fue estudiar la historia de pintado de Oyola 34 para aportar al conocimiento de los diversos procesos de uso, producción y transformación de los abrigos con arte rupestre del sitio arqueológico de Oyola. En pos de alcanzarlo, se plantearon tres objetivos específicos. Primero, acceder a los distintos eventos de confección involucrados en la transformación de las paredes pintadas de la cueva. Luego, indagar en las lógicas que guiaron el agregado de motivos en cada uno de estos eventos. Y, por último, establecer las implicancias e interrelaciones que los cambios en los paneles rupestres pudieron haber tenido con las prácticas sociales desarrolladas en relación con el arte rupestre del abrigo.

\section{Enfoque teórico y metodológico}

Tanto el problema como los objetivos delineados suponen un abordaje material e histórico del arte rupestre. El primer aspecto refiere a que esta tesis se enmarca dentro de un 
Ol: 10.34096/arqueologia t27 n1.9775

ISSN 0327-5159 (impresa) / ISSN 1853-8126 (en línea)

Arqueología 27(1) enero-abril: 209-212 (2021)

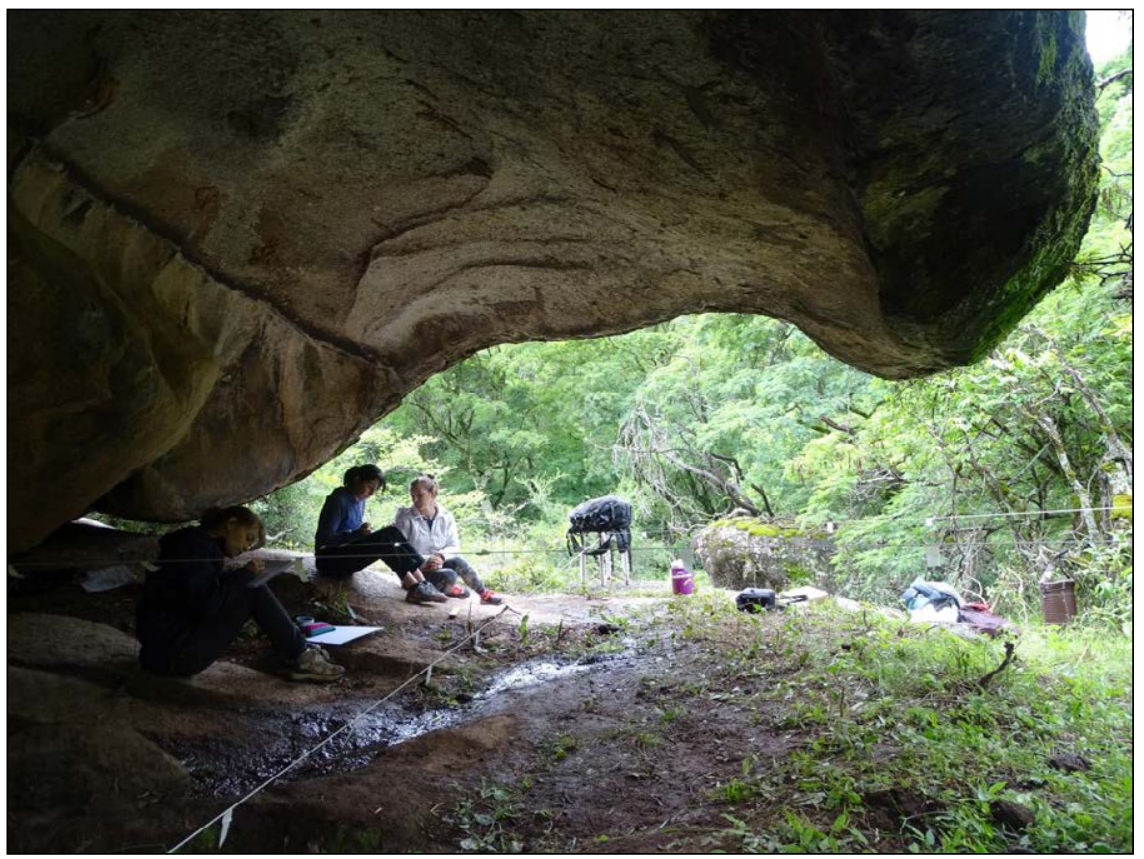

Figura 1. Vista general de la cámara principal de Oyola 34.

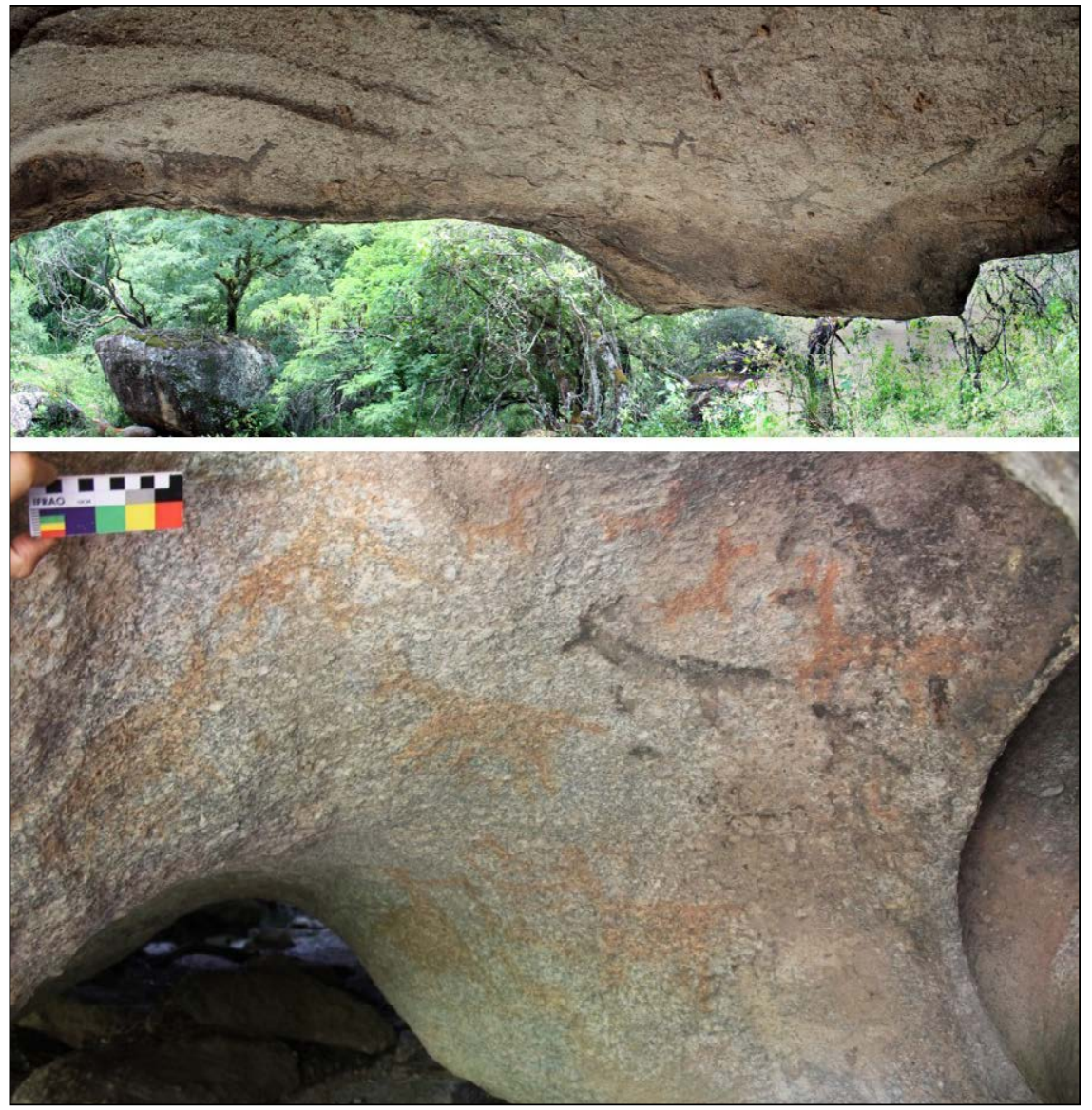

Figura 2. Algunos de los motivos rupestres de Oyola 34. 
conjunto de investigaciones que, a diferencia de otros trabajos que se centran en sus dimensiones ideales o simbólicas, conciben a las pinturas rupestres en tanto realidad material (Aschero, 1988; Fiore, 1996). En esta línea, las preguntas formuladas apuntaron a comprender las prácticas sociales que produjeron las mezclas pigmentarias, modificaron los paneles rupestres y se relacionaron con los motivos pintados en la cueva a lo largo del tiempo. Así, este punto se encuentra estrechamente vinculada con el segundo eje teórico: el arte rupestre como fenómeno histórico, es decir, como producto y, a la vez, agente activo en los procesos históricos a través de los cuales las personas -en su interrelación con los restantes sujetos/objetos- producen y transforman la realidad social (Moore, 2008; Pauketat, 2001). Además, por su carácter perdurable y capacidad de aditividad (Aschero, 1997), los paneles rupestres adquieren un carácter inacabado y pueden pensarse como campos de posibilidades para quienes ejecutaron los motivos y quienes los apreciaron (Martel, Rodríguez Curletto y Del Bell, 2012; Quesada y Gheco, 2015). En definitiva, estas posturas implicaron comprender las particularidades de cada conjunto pictórico a la luz de las sucesivas y, posiblemente, cambiantes prácticas sociales que los ejecutaron, modificaron, usaron, significaron y que, a su vez, se vieron afectadas por ellos a lo largo del tiempo.

Tales consideraciones teóricas también fueron fundamentales para definir la estrategia metodológica implementada. Es así que se adoptó una micro-escala de análisis (Troncoso, 2008) para generar datos de alta resolución y realizar inferencias sobre el accionar situado, estructurado y estructurante, de las personas y las pinturas en Oyola 34. La metodología seleccionada implicó la combinación de múltiples líneas de evidencia complementarias en dos etapas de análisis. En la primera etapa, macroscópica, se realizaron estudios tonales, morfológicos, análisis de las superposiciones entre motivos y de su estructuración espacial (Aschero, 1997; Gheco, 2017; Gradin, 1978; Troncoso, 2008). La segunda etapa, microscópica, incluyó el estudio arqueométrico de muestras microestratigráficas ( $1 \mathrm{~mm}^{2}$ aproximadamente) de algunas de las pinturas empleando dos técnicas de análisis químico (Microscopía electrónica de barrido con análisis elemental por espectroscopía dispersiva de rayos $\mathrm{X}$ y microEspectroscopía Raman) (Gheco et al., 2019; Marte, Mastrangelo y Tascón, 2011).

\section{Resultados}

El estudio de las tonalidades, combinado con los análisis arqueométricos y el relevamiento de las superposiciones permitieron identificar y secuenciar los distintos eventos de confección involucrados en la transformación de las paredes pintadas de la cueva. Por su parte, las características morfológicas de las figuras y sus superposiciones resultaron informativas con respecto a las lógicas que guiaron estos procesos de agregado de motivos. Finalmente, los análisis espaciales (que consideraron el emplazamiento de las pinturas rupestres y sus condiciones de visibilidad) brindaron información acerca de la diversidad de experiencias de observación/acción habilitadas y alentadas por los paneles rupestres. Además, en combinación con los otros resultados, fue posible comprender en perspectiva diacrónica cómo dichas experiencias espaciales cambiaron a lo largo del proceso de pintado de la cueva. De esta forma, las cinco líneas de evidencia consideradas en conjunto y de manera complementaria permitieron comenzar a delinear cómo fue la historia de pintado y uso del abrigo (Ahets Etcheberry y Gheco, en prensa). Para dar cuenta de ella resultó operativo acuñar el concepto de ciclo de pintado -adaptando al estudio del arte rupestre el concepto de ciclo estratigráfico definido por Carandini (1997)- para referir a conjuntos de motivos realizados en un mismo abrigo en un lapso temporal relativamente acotado, cuyos rasgos similares (relativos a las morfologías, colores y disposiciones espaciales) permiten entrever continuidades en las prácticas sociales asociadas a ellos.

El primero y más antiguo de los ciclos de pintado de Oyola 34 involucró la confección de camélidos naranjas empleando diferentes preparaciones de pinturas, tal como sugieren los resultados de los estudios tonales y arqueométricos. Con respecto a estos últimos, en las muestras analizadas se detectó el uso de hematita como pigmento, en ciertos casos combinada con yeso, en otros con yeso y alguna arcilla y en otros con yeso y moscovita. Los motivos se ejecutaron yuxtapuestos, con morfologías y colores similares, de modo que conformaron paneles rupestres relativamente uniformes. Asimismo, este ciclo se vinculó con experiencias de observación de las pinturas rupestres en las que pueden participar pocas personas en simultáneo, en actitudes estáticas y próximas a las paredes. En conjunto, estos aspectos se asemejan a lo identificado en la mayoría de los abrigos del sitio.

Durante el segundo ciclo de pintado se agregaron motivos negros $y$, con ellos, se introdujeron ciertos cambios: superposiciones, nuevos colores, nuevos materiales (los análisis químicos señalan que se emplearon como pigmentos óxidos de manganeso y posiblemente carbón), nuevas morfologías, nuevas técnicas de confección y nuevos emplazamientos. Resulta llamativa la mayor diversidad morfológica observada entre las figuras de este ciclo. Si bien los referentes continúan siendo mayoritariamente camélidos, muchos de ellos presentan características morfológicas innovadoras. Además, en este ciclo se ejecutaron figuras con nuevos referentes (como cérvidos) que no fueron relevados en otros abrigos del sitio. Por último, por su emplazamiento en otros sectores de la cueva, estos motivos debieron impulsar experiencias de observación que habrían involucrado a un mayor número de personas en actitudes dinámicas. En definitiva, este segundo ciclo de pintado da cuenta de cambios en la historia de Oyola 34 que resultan más evidentes y que señalan la excepcionalidad del arte rupestre del abrigo en comparación con el resto de las cuevas del sitio. 
Por último, el tercer ciclo de pintado involucró la ejecución de un escrito en tiza moderno, superpuesto completamente a dos motivos negros del segundo ciclo, generando así una ruptura con los colores, materiales, técnicas y formas previas. Por contraste, esto permite matizar las transformaciones introducidas en el abrigo durante el segundo ciclo de pintado y destacar las continuidades entre ambos ciclos prehispánicos.

En síntesis, los resultados de esta tesis permiten conocer algunos fragmentos de la historia de Oyola 34, cuyas características se condicen, en cierta medida, con las interpretaciones elaboradas para comprender el arte rupestre del sitio. Como vimos, el proceso de agregado de motivos durante el primer ciclo siguió una lógica muy similar a aquella detectada en otras cuevas y aleros. Sin embargo, los principales cambios se dieron durante el segundo y tercer ciclo, rompiendo con el aspecto inalterado de los paneles rupestres que primaba hasta entonces. Ahora bien, a pesar de esto, también fue posible rastrear algunas similitudes entre los ciclos prehispánicos que hacen que, aunque bajo una forma diferente, la historia de pintado de la cueva se aproxime también al estilo de las narrativas de apariencia prístina elaboradas en los restantes abrigos del sitio.

\section{Referencias citadas}

》 Ahets Etcheberry, E. y Gheco, L. (en prensa). Una propuesta metodológica para acceder a las historias de pintado de Oyola 34 (Catamarca). Cuadernos del Instituto Nacional de Antropología y Pensamiento Latinoamericano.

"Aschero, C. A. (1988). Pinturas rupestres, actividades y recursos naturales: un encuadre arqueológico. En $H$. D. Yacobaccio, L. A. Borrero, L. C. García, G. G. Politis, C. A. Aschero y C. Bellelli (Eds.), Arqueología Contemporánea Argentina. Actualidad y Perspectivas (pp. 109-142). Buenos Aires: Ediciones Búsqueda.

»Aschero, C. A. (1997). De cómo interactúan emplazamientos, conjuntos y temas. En Actas y Memorias del XI Congreso Nacional de Arqueología Argentina, Tomo XIII (pp. 17-28). Mendoza: Facultad de Filosofía y Letras, Universidad Nacional de Cuyo.

"Carandini, A. (1997). Historias en la tierra. Manual de excavación. Barcelona: Crítica.

»De la Fuente, N. (1979). Arte rupestre en la región de Ancasti, Prov. de Catamarca. Jornadas de Arqueología de NOA. Antíquitas, 2, 408-418.

"Fiore, D. (1996). El arte rupestre como producto complejo de procesos económicos e ideológicos: una propuesta de análisis. Espacio, Tiempo y Forma, Serie I, Prehistoria y Arqueología, 9, 239-259.

"Gheco, L. (2017). El laberinto de las paredes pintadas. Una historia de los abrigos con Arte Rupestre de Oyola,
Catamarca. (Tesis de Doctorado inédita), Universidad Nacional de Córdoba, Argentina.

» Gheco, L., Tascón, M., Gastaldi, M., Ahets Etcheberry, E., Mastrangelo, N., Quesada, M. y Marte, F. (2019). Hidden paintings, forgotten histories: a micro-stratigraphic approach to study coated rock art. Archaeological and Anthropological Sciences, 11, 5037-5052. https://doi. org/10.1007/s12520-019-00854-z

»González, A. R. (1977). Arte Precolombino de la Argentina. Buenos Aires: Filmediciones Valero.

» Gordillo, I., Zuccarelli, V. N. y Eguía, L. (2017). Las casas del sol naciente: Arqueología de la vertiente oriental de El Alto-Ancasti. En M. F. Becerra, B. N. Ventura, G. Ortiz y M. B. Cremonte (Eds.), Arqueología de la vertiente oriental Surandina: interacción macro-regional, materialidades, economía y ritualidad (pp. 111-134). Buenos Aires: Sociedad Argentina de Antropología.

»Gradin, C. (1978). Algunos aspectos del análisis de las manifestaciones rupestres. Revista del Museo Provincial. Arqueología, 1, 120-133.

» Marte, F., Mastrangelo, N. y Tascón, M. (2011). El arte de medir: microscopía óptica aplicada a la medición de secciones transversales. Eadem utraque Europa, 7(13), 257-268.

» Martel, Á., Rodríguez Curletto, S. y Del Bell, E. (2012). Arte rupestre y espacios de memoria: Las representaciones del sitio Confluencia (Antofagasta de la Sierra, Catamarca, Argentina). Revista de Antropología, 25(1), 121-162.

" Moore, J. (2008). Making a huaca. Memory and praxis in prehispanic far northern Peru. Journal of Social Archaeology, 10(3), 398-422. https://doi. org $/ 10.1177 / 1469605310381550$

" Pauketat, T. (2001). Practice and history in archaeology. Anthropological Theory, 1, 73-98. https://doi. org/10.1177/146349960100100105

»Quesada, M., Gastaldi, M. y Granizo, G. (2012). Construcción de periferias y producción de lo local en las cumbres del Alto-Ancasti. Relaciones de la Sociedad Argentina de Antropología, XXXVII(2), 435-456.

»Quesada, M. y Gheco, L. (2015). Tiempos, cuevas y pinturas. Reflexiones sobre la policronía del arte rupestre de Oyola (Provincia de Catamarca, Argentina). Relaciones de la Sociedad Argentina de Antropología, XL(2), 455-476.

"Segura, A. (1988). El Arte Rupestre del Este de Catamarca. Las Pictografías de la Candelaria. Dpto. Ancasti, Provincia de Catamarca. San Fernando del Valle de Catamarca: Editorial Universitaria. Facultad de Humanidades. Universidad Nacional de Catamarca.

"Troncoso, A. (2008). Arte rupestre y microespacios en el valle de Putaendo, Chile: entre la movilidad, la visibilidad y el sentido. En A. Nielsen, C. Rivolta, V. Seldes y M. Vásquez (Eds.), Procesos Sociales en los Andes Meridionales (pp. 393-410). Córdoba: Editorial Brujas. 